\title{
Qualidade da alimentação de idosos longevos e doenças crônicas não transmissíveis
}

\section{Food quality of long elderly and non-communicable chronic diseases}

\author{
Ana Cristina Martins Passos ${ }^{1}$, Dalila Pinto de Souza Fernandes ${ }^{2}$, \\ Andréia Queiroz Ribeiro ${ }^{3}$, Regina Célia Rodrigues de \\ Miranda Milagres ${ }^{4}$, Maria Sônia Lopes Duarte ${ }^{5}$
}

\section{Resumo}

Avaliou-se a qualidade da alimentação de idosos longevos e sua relação com doenças crônicas não transmissíveis. Foi um estudo transversal, de base populacional, realizado em Viçosa, Minas Gerais, Brasil, o qual integra um projeto maior intitulado "Condições de saúde, nutrição e uso de medicamentos por idosos do município de Viçosa (MG): um inquérito de base populacional”. As entrevistas com os idosos foram realizadas por meio de questionário semiestruturado, com a maioria das questões fechadas e pré-codificadas. Os dados do consumo alimentar foram obtidos por recordatório de ingestão habitual e a qualidade da dieta avaliada por meio do Índice de Alimentação Saudável Revisado (IAS-R), validado para a população brasileira. A amostra foi composta por 94 idosos longevos, a maioria do sexo feminino. Os mesmos apresentaram alta ingestão de sódio, baixa ingestão de cereais integrais e IAS-R $=62,22$. Idosos diabéticos apresentaram maior ingestão de frutas totais, baixa ingestão de Gord_AA (açúcares, gorduras sólidas e álcool) e IAS-R total maior em comparação aos não diabéticos. As presenças de dislipidemia e hipertensão não alteraram o consumo de nenhum dos componentes. Os resultados indicaram a necessidade de adequação na ingestão alimentar. Assim, intervenções e orientações nutricionais específicas destinadas à promoção da saúde devem ser incentivadas.

Palavras-chave: Saúde do idoso; Consumo alimentar; Nutrição do idoso.

${ }^{1}$ Graduação em Nutrição pela Universidade Federal de Viçosa (UFV), Viçosa, Minas Gerais, Brasil.

${ }^{2}$ Doutorado em Ciência da Nutrição pela Universidade Federal de Viçosa, Viçosa, Minas Gerais, Brasil.

${ }^{3}$ Doutorado em Ciências Farmacêuticas pela Universidade Federal de Minas Gerais (UFMG), Belo Horizonte, Minas Gerais, Brasil. Professora Associada do Departamento de Nutrição e Saúde da Universidade Federal de Viçosa, Viçosa, Minas Gerais, Brasil.

${ }^{4}$ Doutorado em Ciências pelo Centro de Energia Nuclear na Agricultura da Universidade de São Paulo (CENA/USP), Piracicaba, São Paulo, Brasil. Técnica de Nível Superior do Departamento de Nutrição e Saúde da Universidade Federal de Viçosa, Viçosa, Minas Gerais, Brasil.E-mail: reginamilagres@ufv.br

${ }^{5}$ Doutorado em Ciência e Tecnologia de Alimentos pela Universidade Federal de Viçosa, Viçosa, Minas Gerais, Brasil. Professora Associada do Departamento de Nutrição e Saúde da Universidade Federal de Viçosa, Viçosa, Minas Gerais, Brasil. 


\begin{abstract}
The quality of the food of long-lived elderly and its relationship with non-communicable chronic diseases was evaluated. This a cross-sectional study with a population basis that was conducted in Viçosa, Minas Gerais, Brazil, which is part of a larger project entitled "Health, nutrition and drug use by older people in Viçosa (MG): a population-based survey". The interviews with the elderly was conducted through a semi-structured questionnaire, with most questions closed and pre-coded. Food intake data were obtained from the usual intake recall and the quality of the diet was assessed using the Revised Healthy Eating Index (HEI-R), validated for the Brazilian population. The sample consisted of 94 long-lived elderly, most of them female. They presented high sodium intake, low whole grains intake and HEI-R $=62.22$. Diabetic older adults had higher total fruit intake, lower intake of Gord_AA (sugars, solid fats, and alcohol) and higher total HEI-R compared to non-diabetics. The presences of dyslipidemia and hypertension did not alter the consumption of any of the components. The results indicated the need for adequacy in food intake. Thus, specific nutritional interventions and guidelines for health promotion should be encouraged.
\end{abstract}

Keywords: Health of the elderly; Food consumption; Elderly nutrition.

\section{Introdução}

O envelhecimento populacional é atualmente reconhecido como fenômeno característico tanto dos países desenvolvidos como dos países em desenvolvimento, sendo que a faixa etária mais crescente no mundo é a idosos longevos, aqueles que têm 80 anos ou mais. ${ }^{(1)}$ As projeções indicam que no Brasil, em 2050, ocorrerá um aumento desse grupo para 13,8 milhões, ${ }^{(2)}$ fato que aponta para implicações na área de saúde, já que a prevalência de morbidades e incapacidades será maior. ${ }^{(3)}$

Estima-se que as doenças crônicas não transmissíveis (DCNT) serão mais constantes nos idosos de 80 anos ou mais, ${ }^{(4)}$ o que contribuirá para o aparecimento de dificuldades nas atividades habituais com interferência na sua independência e autonomia. ${ }^{(5)}$ Neste contexto, os idosos longevos apresentam características diferentes de outros indivíduos, como maior declínio da capacidade funcional e um número crescente de DCNT. Estima-se que $85 \%$ dos idosos com 80 anos ou mais têm pelo menos uma doença diagnosticada e $50,4 \%$ têm duas doenças. ${ }^{(6)}$

Dentre os fatores de risco para tais doenças, a importância da alimentação é evidenciada em diferentes estudos epidemiológicos ${ }^{(7)} \mathrm{e}$ o hábito alimentar inadequado é apontado como um dos principais fatores de risco. ${ }^{(8)}$

Neste contexto, avaliar a qualidade da dieta de idosos longevos é relevante, já que a alimentação adequada é essencial no tratamento e prevenção das DCNT. ${ }^{(9)}$ Algumas ferramentas têm sido desenvolvidas para viabilizar essa avaliação, dentre elas os índices dietéticos, construídos a partir dos guias alimentares populacionais. Estes consideram o número de porções consumidas, a variedade da alimentação e a adequação da ingestão de nutrientes para avaliar a ingestão alimentar. ${ }^{(10-13)} \mathrm{O}$ Índice de Alimentação Saudável desenvolvido por Kennedy et $a l .{ }^{(11)}$ é uma ferramenta considerada adequada para avaliar a qualidade da dieta e foi atualizado por Guenther, Reedy e Krebs-Smith. ${ }^{(14)}$ No Brasil este índice foi revisado por Previdelli et al. ${ }^{(15)} \mathrm{e}$ validado por Andrade et al. ${ }^{(13)}$ para a população brasileira, denominado Índice de Alimentação Saudável Revisado (IAS-R). No entanto, ainda são escassos estudos com idosos longevos, que permitam o conhecimento das condições de saúde dessa população.

A partir do exposto, o objetivo do estudo foi avaliar a qualidade da dieta de idosos longevos por meio do IAS-R e verificar sua relação com as DCNT. 


\section{Métodos}

\section{Delineamento do estudo e população-alvo}

Estudo transversal, de base populacional, realizado em Viçosa, Minas Gerais, Brasil, o qual integra um projeto maior intitulado "Condições de saúde, nutrição e uso de medicamentos por idosos do município de Viçosa ( $\mathrm{MG}$ ): um inquérito de base populacional", conforme metodologia descrita por Nascimento et al. ${ }^{(16)}$

A população-alvo do estudo foi obtida a partir de um total de 7.980 idosos com 60 anos ou mais, não institucionalizados, residentes nas zonas rural e urbana do município. Para o presente estudo, foram incluídos os idosos longevos (80 anos ou mais), os quais totalizaram 94. A seleção foi feita por amostragem aleatória simples. ${ }^{(17)} \mathrm{O}$ estudo foi aprovado pelo Comitê de Ética em Pesquisa com Seres Humanos da Universidade Federal de Viçosa (UFV) sob parecer número 027/2008.

\section{Coleta de dados}

O primeiro contato foi realizado por telefone ou visita domiciliar para informar os objetivos da pesquisa, convidar o idoso a participar e agendar a entrevista, que foi domiciliar e conduzida por cinco duplas de entrevistadores. Foram realizadas até três tentativas de visita ao domicílio para localização do idoso antes de se considerar como perda.

As entrevistas ocorreram no período de junho a dezembro do ano de 2009, a partir de questionário semiestruturado, com a maioria das questões fechadas e pré-codificadas, testado em estudo piloto. As perguntas foram feitas diretamente aos idosos, mas nos casos em que eles apresentaram dificuldades para responder, um parente ou cuidador os auxiliaram.

Foram coletadas as variáveis sexo, idade, escolaridade e renda individual (ocupação remunerada, aposentadoria e/ou pensão, ajuda financeira). A respeito do histórico de DCNT, foram incluídas neste estudo diabetes mellitus, hipertensão e dislipidemia. Estas doenças foram coletadas a partir da resposta à seguinte pergunta: "alguma vez na vida um médico ou outro profissional de saúde disse que o(a) Sr.(a.) tem ou teve alguma das seguintes doenças?".

Os dados do consumo alimentar foram obtidos a partir da aplicação de um recordatório de ingestão habitual, com utilização do método de passagens múltiplas. Os detalhes metodológicos podem ser vistos em outra publicação. ${ }^{(18)} \mathrm{O}$ cálculo da estimativa do consumo alimentar e do valor de macronutrientes e micronutrientes dos alimentos consumidos foi realizado com auxílio do software Dietpro $^{\circledR}$ versão 5 i.

A partir das informações obtidas no recordatório foram padronizadas as preparações, bem como os percentuais de sal e óleo. ${ }^{(19-20)}$ Foram considerados açúcares de adição todos os açúcares adicionados aos alimentos durante o seu processamento, preparo ou consumo. A quantidade de açúcar de adição presente em cada alimento foi padronizada com base na Tabela para avaliação do consumo alimentar em medidas caseiras, ${ }^{(19)} \mathrm{em}$ receitas e no rótulo dos alimentos.

\section{Índice de Alimentação Saudável Revisado}

A qualidade da dieta foi avaliada por meio do Índice de Alimentação Saudável Revisado para a população brasileira (IAS-R), proposto por Previdelli et al. ${ }^{(15)}$ e validado por Andrade et al. ${ }^{(13)}$ (Tabela 1).

Este índice é composto por 12 componentes baseados em grupos de alimentos (Frutas totais; Frutas integrais; Cereais totais; Cereais integrais; Carnes, ovos e leguminosas; Vegetais totais; Vegetais verde-escuros e alaranjados e leguminosas; Leite e derivados; e Óleos), nutrientes (Gordura saturada e Sódio) e a soma do valor energético proveniente da ingestão de gorduras sólidas (saturada e trans), álcool e açúcar de adição (Gord_AA).

Para o cálculo da pontuação de cada componente estimou-se o número de porções consumidas por $1.000 \mathrm{kcal}$ para os grupos de alimentos, em mg/1.000 kcal para sódio e proporção do consumo energético total pelo nutriente para gordura 
saturada e gorduras sólidas, açúcar e álcool (Gord AA), sendo o valor máximo de 100 pontos para o IAS-R total. Em relação aos componentes individuais, as pontuações variam de zero (mínima) a 5, a 10 ou a 20 (máximas), dependendo do componente. A pontuação mínima refere-se ao consumo nulo (componentes 1 a 9) ou ao consumo acima do limite preconizado (componentes 10 a 12), e a pontuação máxima é estabelecida atingindo ou ultrapassando o valor recomendado de ingestão (componentes 1 a 9). Para os componentes Gordura saturada, Sódio e Gord_AA a pontuação é inversa, pois quanto maior o consumo, menor a pontuação, visto que são considerados componentes de moderação, ou seja, sua ingestão em excesso deve ser evitada. $^{(12,15)}$

Tabela 1 - Distribuição da pontuação e das porções dos componentes do Índice de Alimentação Saudável Revisado (IAS-R).

\begin{tabular}{|c|c|c|c|c|}
\hline \multirow{2}{*}{ Componentes } & \multicolumn{4}{|c|}{ Pontuação (pontos) } \\
\hline & $\mathbf{0}$ & 5 & 10 & 20 \\
\hline 1. Frutas totais ${ }^{\text {a }}$ & 0 & 1,0 porção/1.000 kcal & & \\
\hline 2. Frutas integrais ${ }^{b}$ & 0 & 0,5 porção/1.000 kcal & & \\
\hline 3. Vegetais totais ${ }^{c}$ & 0 & 1,0 porção/1.000 kcal & & \\
\hline 4. Veveal ${ }^{\mathrm{d}}$ & 0 & 0,5 porção/1.000 kcal & & \\
\hline 5. Cereais totais ${ }^{e}$ & 0 & & & \\
\hline 6. Cereais integrais & 0 & 2,0 porções $/ 1.000 \mathrm{kcal}$ & & \\
\hline 7. Leite e derivados ${ }^{f}$ & 0 & & 1,5 porção/1.000 kcal & \\
\hline 8. Carnes, ovos e leguminosas & 0 & & 1,0 porção/1.000 kcal & \\
\hline 9. Óleos ${ }^{\mathrm{g}}$ & 0 & & 0,5 porção $/ 1.000 \mathrm{kcal}$ & \\
\hline 10. Gordura saturada & $\geq 15$ & & $\leq 7 \%$ do VET & \\
\hline 11. Sódio & $\geq 2,0$ & & $\leq 0,75 \mathrm{~g} / 1.000 \mathrm{kcal}$ & \\
\hline 12. Gord_AA ${ }^{\mathrm{h}}$ & $\geq 35$ & & & $\leq 10 \%$ do VET \\
\hline
\end{tabular}

Fonte: Andrade et al. ${ }^{(13)}$

a Inclui frutas e sucos de frutas naturais.

${ }^{\mathrm{b}}$ Exclui sucos de frutas.

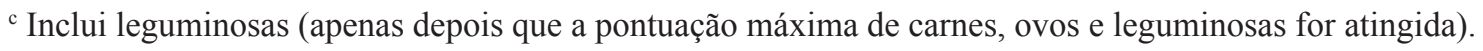

${ }^{\mathrm{d}}$ Veveal: Vegetais verde-escuros e alaranjados e leguminosas (apenas depois que a pontuação máxima de carnes, ovos e leguminosas for atingida).

${ }^{\text {e }}$ Cereais totais: representa o grupo de cereais, raízes e tubérculos.

${ }^{\mathrm{f}}$ Inclui leite e derivados e bebidas à base de soja.

${ }^{g}$ Inclui as gorduras monoinsaturadas e poli-insaturadas, oleaginosas e gordura de peixe.

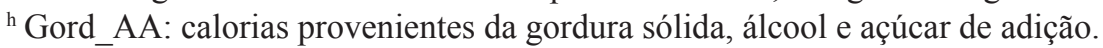

VET: valor energético total.

Estimou-se a pontuação do componente Carne, ovos e leguminosas a partir do somatório do valor energético do grupo Carnes e ovos, e Leguminosas. Caso houvesse calorias excedentes, o valor calórico proveniente das Leguminosas foi computado nos grupos Vegetais verde-escuros e alaranjados e leguminosas (Veveal) e Vegetais totais, simultaneamente.

Ao avaliar a pontuação total, os valores mais próximos da pontuação máxima indicam melhor 
qualidade da dieta. Como o índice pode refletir diferentes aspectos da dieta, deve-se avaliar a pontuação de cada componente isoladamente..$^{(12,14)}$

\section{Análise dos dados}

Foi realizada a análise descritiva dos dados, com distribuição de frequências (variáveis qualitativas) e estimação de medidas de tendência central e de dispersão (variáveis quantitativas). A avaliação da normalidade das variáveis dentro dos grupos de comparação foi realizada pelo teste de Kolmogorov-Smirnov.

Como as variáveis apresentaram distribuição assimétrica, foi utilizado o teste Mann Whitney para comparação da média da pontuação do IAS-R total e dos componentes de acordo com as DCNT. Todas as análises foram realizadas com auxílio do software SPSS ${ }^{\circledR}$ versão 20.0 (SPSS Inc., Chicago, Illinois, Estados Unidos), adotando-se como nível de significância estatística $\alpha=0,05$ para todas as comparações.

\section{Resultados}

A amostra estudada consistiu de 94 idosos. De acordo com as características socioeconômicas, a maioria era do sexo feminino $(64,9 \%)$, com idade média de $85 \pm 4,4$ anos e escolaridade até o ensino fundamental I completo ou incompleto. Cerca de $60 \%$ tinham renda per capita entre R\$465,00 e $\mathrm{R} \$ 522,49$ (segundo quartil/Q2), considerando que o limite inferior corresponde ao valor do salário mínimo da época do estudo. Cerca de $27 \%$ tinham histórico de diabetes mellitus, $80 \%$ de hipertensão arterial e $51 \%$ de dislipidemia (Tabela 2).

Tabela 2 - Características socioeconômicas e de saúde de idosos longevos, Viçosa (MG), 2009.

\begin{tabular}{lc}
\hline Variáveis & Frequência: N (\%) \\
\hline Sexo & $33(35,1)$ \\
Homens & $61(64,9)$ \\
Mulheres & \\
Escolaridade & $27(28,7)$ \\
Nunca estudou & $58(61,7)$ \\
Ensino fundamental I completo ou incompleto & $9(9,6)$ \\
Ensino fundamental II completo ou mais & \\
Renda individual (quartis) & $4(4,4)$ \\
Q1 (R\$ 0 - R\$ 464,99) & $57(60,4)$ \\
Q2 (R 465,00 - R\$ 522,49) & $21(22,0)$ \\
Q3 (R\$ 522,50 - R\$ 1.476,24) & $12(13,2)$ \\
Q4 ( $\geq$ R $1.476,25)$ & \\
Histórico de diabetes mellitus & $25(26,6)$ \\
Sim & $69(73,4)$ \\
Não & \\
Histórico de hipertensão & $75(79,8)$ \\
Sim & $19(20,2)$ \\
Não &
\end{tabular}

Fonte: dados da pesquisa. 
A média da pontuação do IAS-R total foi maiores foram Vegetais verde-escuros e alaranjade 62,22 $\pm 11,62$. Os componentes com menores dos; Vegetais totais; Cereais totais; Carnes, ovos e percentuais foram Sódio e Cereais integrais, e com leguminosas; Óleos e Gordura saturada (Tabela 3).

Tabela 3 - Valores médios e relação entre a média e a pontuação máxima de cada componente do Índice de Alimentação Saudável Revisado (IAS-R) de idosos longevos, Viçosa (MG).

\section{Componente do IAS-R}

\section{Pontuações}

0 a 5

0 a 5

0 a 5

0 a 5

0 a 5

0 a 5

0 a 10

0 a 10

0 a 10

0 a 10

0 a 10

0 a 20

Gord_AA ${ }^{4}$

IAS-R total

Gordura saturada

Sódio

Carnes, ovos e leguminosas

Óleos
Média \pm DP $^{1}$

$\mathbf{M} / \mathbf{P}_{\max }{ }^{2}(\%)$

$2,77 \pm 2,23$

55,4

$2,87 \pm 2,42$

57,4

91,4

$4,57 \pm 1,07$

93,0

$4,65 \pm 1,09$

93,4

$4,67 \pm 0,74$

13,2

$0,66 \pm 1,47$

51,5

$5,15 \pm 3,86$

92,0

$9,20 \pm 1,66$

79,5

$7,95 \pm 2,52$

84,9

$8,49 \pm 2,59$

10,3

$1,03 \pm 1,93$

$10,01 \pm 7,05$

50,0

$62,22 \pm 11,62$

Fonte: dados da pesquisa.

${ }^{1}$ DP: desvio-padrão.

${ }^{2} \mathrm{M} / \mathrm{P}_{\max }$ : relação entre a média e a pontuação máxima.

${ }^{3}$ Veveal: Vegetais verde-escuros e alaranjados e leguminosas.

${ }^{4}$ Gord_AA: gorduras sólidas (saturada e trans), álcool e açúcar de adição.

Idosos longevos com histórico de diabetes mellitus apresentaram pontuações maiores para Frutas totais, Gord_AA e IAS-R total quando comparados aos não diabéticos. Entre os idosos com dislipidemia e hipertensão não houve diferença entre o IAS-R e seus componentes em relação aos que não apresentavam histórico para essas doenças (Tabela 4). 
Tabela 4 - Média e desvio-padrão da pontuação total dos componentes do Índice de Alimentação Saudável Revisado (IAS-R) segundo doenças crônicas não transmissíveis de idosos longevos, Viçosa (MG), 2009.

\begin{tabular}{|c|c|c|c|c|c|c|c|c|c|c|c|c|c|}
\hline Doenças & $\begin{array}{c}\text { Frutas } \\
\text { totais }\end{array}$ & $\begin{array}{l}\text { Frutas } \\
\text { integrais }\end{array}$ & $\begin{array}{c}\text { Vegetais } \\
\text { totais }\end{array}$ & Veveal $^{1}$ & $\begin{array}{c}\text { Cereais } \\
\text { totais }\end{array}$ & $\begin{array}{c}\text { Cereais } \\
\text { integrais }\end{array}$ & $\begin{array}{c}\text { Leite e } \\
\text { derivados }\end{array}$ & $\begin{array}{c}\text { Carnes, } \\
\text { ovos e } \\
\text { leguminosas }\end{array}$ & Óleos & $\begin{array}{l}\text { Gordura } \\
\text { saturada }\end{array}$ & Sódio & Gord_AA ${ }^{2}$ & IAS- $\mathbf{R}^{3}$ \\
\hline \multicolumn{14}{|l|}{ Diabetes } \\
\hline Sim & $3,86 \pm 2,00$ & $3,55 \pm 2,27$ & $4,42 \pm 1,19$ & $4,28 \pm 1,6$ & $4,69 \pm 0,85$ & $0,66 \pm 1,38$ & $4,87 \pm 3,93$ & $9,42 \pm 1,37$ & $8,47 \pm 2,18$ & $9,33 \pm 1,32$ & $0,68 \pm 1,48$ & $15,53 \pm 6,49$ & $69,82 \pm 12,01$ \\
\hline Não & $2,37 \pm 2,18$ & $2,62 \pm 2,43$ & $4,62 \pm 1,03$ & $4,78 \pm 0,78$ & $4,66 \pm 0,70$ & $0,66 \pm 1,51$ & $5,25 \pm 3,86$ & $9,12 \pm 1,75$ & $7,76 \pm 2,62$ & $8,18 \pm 2,87$ & $1,16 \pm 2,06$ & $8,01 \pm 6,16$ & $59,46 \pm 10,23$ \\
\hline $\mathrm{P}$ & $* 0,020$ & 0,098 & 0,296 & 0,168 & 0,4 & 0,78 & 0,704 & 0,35 & 0,181 & 0,07 & 0,57 & $* 0,000$ & $* 0,000$ \\
\hline \multicolumn{14}{|c|}{ Dislipidemia } \\
\hline Sim & $2,92 \pm 2,29$ & $2,96 \pm 2,43$ & $4,67 \pm 0,82$ & $4,74 \pm 0,86$ & $4,67 \pm 0,83$ & $0,72 \pm 1,62$ & $5,37 \pm 3,72$ & $9,38 \pm 1,38$ & $7,61 \pm 2,52$ & $8,36 \pm 2,82$ & $0,94 \pm 1,74$ & $10,44 \pm 7,36$ & $62,66 \pm 12,44$ \\
\hline Não & $2,60 \pm 2,17$ & $2,76 \pm 2,42$ & $4,45 \pm 1,28$ & $4,56 \pm 1,30$ & $4,67 \pm 0,64$ & $0,59 \pm 1,31$ & $4,92 \pm 4,02$ & $9,02 \pm 1,90$ & $8,31 \pm 2,49$ & $8,62 \pm 2,35$ & $1,12 \pm 2,12$ & $9,56 \pm 6,77$ & $61,75 \pm 10,82$ \\
\hline $\mathrm{P}$ & 0,419 & 0,721 & 0,764 & 0,855 & 0,558 & 0,722 & 0,796 & 0,457 & 0,166 & 0,587 & 0,816 & 0,455 & 0,607 \\
\hline \multicolumn{14}{|c|}{ Hipertensão } \\
\hline Sim & $2,92 \pm 2,23$ & $2,97 \pm 2,40$ & $4,54 \pm 1,09$ & $4,62 \pm 1,18$ & $4,65 \pm 0,69$ & $0,72 \pm 1,53$ & $5,13 \pm 3,76$ & $9,11 \pm 1,71$ & $8,00 \pm 2,54$ & $8,45 \pm 2,63$ & $1,07 \pm 2,03$ & $10,25 \pm 7,20$ & $62,62 \pm 11,90$ \\
\hline Não & $2,17 \pm 2,16$ & $2,45 \pm 2,50$ & $4,66 \pm 1,02$ & $4,78 \pm 0,68$ & $4,72 \pm 0,92$ & $0,40 \pm 1,16$ & $5,23 \pm 4,33$ & $9,54 \pm 1,44$ & $7,75 \pm 2,48$ & $8,64 \pm 2,52$ & $0,87 \pm 1,46$ & $9,06 \pm 6,53$ & $60,63 \pm 10,65$ \\
\hline $\mathrm{P}$ & 0,214 & 0,608 & 0,437 & 0,721 & 0,396 & 0,551 & 0,824 & 0,338 & 0,399 & 0,945 & 0,656 & 0,577 & 0,443 \\
\hline
\end{tabular}

Fonte: dados da pesquisa.

*Teste Mann Whitney, $\mathrm{p}<0,05$.

${ }^{1}$ Veveal: Vegetais verde-escuros e alaranjados e leguminosas.

${ }^{2}$ Gord_AA: calorias provenientes de gordura sólida, álcool e açúcar de adição.

${ }^{3}$ IAS-R: Índice de Alimentação Saudável Revisado. 


\section{Discussão}

Este estudo foi o primeiro que utilizou o IAS-R para avaliar a qualidade da dieta de idosos longevos. A maioria dos idosos era do sexo feminino, possuía baixa escolaridade e renda per capita no segundo quartil (entre $\mathrm{R} \$ 465,00$ e $\mathrm{R} \$ 522,49$ ), semelhantes a outros estudos. ${ }^{(21-23)}$

Neste estudo os Cereais integrais apresentaram baixa pontuação, resultado semelhante ao encontrado em outros estudos realizados com idosos no Brasil. ${ }^{(24-26)}$ Esse achado pode ser atribuído à dificuldade dos idosos de mastigação e deglutição de alimentos ricos em fibras. ${ }^{(3)} \mathrm{O}$ envelhecimento geralmente vem acompanhado de alterações, como diminuição da capacidade mastigatória, dificuldades de deglutição, diminuição da salivação e modificações no paladar. ${ }^{(27-28)}$ Vale ressaltar que o consumo de cereais integrais possui ligação com a prevenção de doenças cardiovasculares e sua ingestão deve ser estimulada. ${ }^{(29-30)}$

A ingestão de sódio foi alta entre os idosos, o que está de acordo com dados do Instituto Brasileiro de Geografia e Estatística (IBGE). ${ }^{(6)}$ Sabe-se que o envelhecimento gera alterações que refletem na ingestão alimentar; uma delas é a diminuição da sensibilidade. Essas alterações podem favorecer o consumo exagerado de sal, o que pode justificar, em parte, essa alta ingestão. Vale ressaltar que o consumo alto de sal pode contribuir para o surgimento e agravamento de hipertensão na população idosa, ${ }^{(31)}$ o que corrobora os resultados encontrados no presente estudo, que revelou alta prevalência de hipertensão.

No envelhecimento é comum o uso de diversos medicamentos que influenciam na ingestão de alimentos, na digestão, na absorção e na utilização de diversos nutrientes, o que pode comprometer o estado de saúde e a necessidade nutricional do indivíduo idoso. ${ }^{(32)}$ Os poucos estudos sobre alimentação de idosos de forma geral, mas não de idosos longevos, indicam que menos de $10 \%$ dos idosos brasileiros possuem alimentação adequada e que a maioria necessita de modificações para atender suas necessidades nutricionais. ${ }^{(33-34)}$
Para Frutas totais, e integrais, foi observado consumo intermediário, o que está de acordo com o estudo que avaliou 44 idosos com idade igual ou superior a 90 anos, residentes em Porto Alegre, Rio Grande do Sul, Brasil. ${ }^{(35)}$ Há evidências que dietas ricas em frutas e hortaliças contribuem para a proteção à saúde e diminuição do risco de ocorrência de várias doenças; ${ }^{(36)}$ assim, o seu consumo deve ser estimulado nessa população.

Para o componente Leite e derivados foi observada também pontuação intermediária, o que demonstra consumo inadequado. Vale ressaltar que esse componente é a principal fonte de cálcio para idosos. ${ }^{(37)}$

O componente Gord_AA apresentou pontuação intermediária, indicando consumo acima do esperado de açúcares, gorduras sólidas e álcool. Neste estudo os açúcares de adição foram os que mais contribuíram para esse componente. Estes achados são preocupantes, porque os açúcares se relacionam diretamente com as DCNT. ${ }^{(3,38)}$

Em contrapartida, nos grupos de vegetais (Vegetais totais e Vegetais verde-escuros e alaranjados); Cereais totais; Carnes, ovos e leguminosas; Óleos e Gordura saturada o consumo foi adequado, o que deve ser um ponto a ser mantido/reforçado nessa população.

A presença ou ausência de doenças não alterou a pontuação total dos componentes e o IAS-R, com exceção dos idosos diabéticos, que obtiveram maior pontuação para Frutas totais, Gord_AA e IAS-R total quando comparados aos não diabéticos. $\mathrm{O}$ fato de este grupo consumir o componente Frutas totais (suco de frutas + frutas integrais) aponta para maior consumo de suco de frutas nesse grupo, já que para o componente Frutas integrais não houve diferença. A boa pontuação para o componente Gord_AA nos idosos com diabetes é positiva no controle da doença.

Nos idosos que apresentaram dislipidemia e hipertensão não houve alteração no IAS-R e seus componentes, sugerindo que a presença dessas doenças não alterou o hábito alimentar dos idosos longevos. Resultado semelhante foi encontrado por Zanchim et al. ${ }^{(39)}$ 
Dentre as DCNT, a hipertensão foi a mais prevalente neste estudo $(79,8 \%)$, o que reforça os relatos de Cesarino et al. ${ }^{(40)}$ de que a prevalência de HAS em idosos é maior que $60 \%$ e com o avanço da idade os riscos para adquirir hipertensão arterial podem ser maiores. Não foi evidenciada diferença em nenhum dos componentes entre os hipertensos e não hipertensos, ainda que nesta enfermidade seja imprescindível a adoção de estilo de vida saudável, incluindo a redução do consumo de sódio. ${ }^{(41)}$ $\mathrm{O}$ efeito da diminuição da ingestão de sódio no controle da hipertensão e na diminuição do risco de eventos cardiovasculares tende a ser ainda maior em idosos e hipertensos, ${ }^{(30)}$ o que reforça a necessidade de orientações nutricionais direcionadas a essa população. ${ }^{(42)}$

A inadequação da alimentação dos idosos neste estudo (IAS-R = 62,22) somada à prevalência de DCNT, reforça a importância de intervenções com vistas a uma alimentação rica e variada com diversificação dos grupos alimentares para evitar desequilíbrios nutricionais e alcançar maior longevidade e melhor qualidade de vida. ${ }^{(35)}$

\section{Conclusão}

A qualidade da dieta dos idosos longevos precisa de adequação, pois a maior parte dos idosos apresentava hipertensão arterial, alto consumo de sódio e baixo consumo de cereais integrais. A presença de doenças crônicas não transmissíveis não alterou o consumo alimentar dos idosos, exceto para os diabéticos. Diante disso, torna-se evidente a necessidade de acompanhamento nutricional para adequar as práticas alimentares na prevenção e controle de doenças crônicas não transmissíveis.

\section{Referências}

1 Granic A, Mendonça N, Hill TR, Jagger C, Stevenson EJ, Mathers JC, et al. Nutrition in the Very Old. Nutrients. 2018;10(3):269.

2 Instituto Brasileiro de Geografia e Estatística. Projeção da população do Brasil por sexo e idade - 1980-2050. Rio de Janeiro: IBGE; 2008.
3 Duarte MSL, Gomes ECS, Loureiro LMR. Recomendações nutricionais para idosos: abordagem nutricional em idosos. Rio de Janeiro: Editora Rubio; 2016.

4 Paixão JCM, Reichenrein ME. Uma revisão sobre instrumentos da avaliação do estado funcional do idoso. CadSaudePublica 2005; 21(1): 7-19.

5 Lourenço TM, Lenardt $\mathrm{MH}$, Kletemberg DF, Seima MD, Tallmann AEC, Neu DKM. Capacidade funcional no idoso longevo: uma revisão integrativa. Rev Gaúcha Enferm. 2012; 33(2):176-85.

6 Instituto Brasileiro de Geografia e Estatística (IBGE). Censo Demográfico 2010. Rio de Janeiro: IBGE; 2011.

7 Global Burden of Diseases. Injuries, and Risk Factors Study. The Lancet [Internet]. 2012. [cited 2017 Jun 10]. Available from: http:// www.thelancet.com/themed/global-burden-ofdisease

8 Willett WC. Overview of nutritional epidemiology. Nutritional epidemiology. Oxford: Oxford University Prees; 1998.

9 Christensen K, Doblhammer G, Rau R, Vaupel JW. Ageing population: the challenges ahead. Lancet [Internet]. 2009 [cited 2019 Aug 20]; 374(9696):1196-208. Available from: http:/www.ncbi.nlm.nih.gov/pmc/articles/ PMC2810516/pdf/nihms164804.pdf

10 Fisberg RM, Slater B, Barros RR, Lima FD, Cesar CLG, Carandina L, et al. Índice de qualidade da dieta: avaliação da adaptação e aplicabilidade. RevNutr 2004;17(3):301-8.

11. Kennedy ET, Ohls J, Carlson S, Fleming K. The healthy eating index: desingand applications. J Am Diet Assoc 1995;95(10):1108-13.

12 Guenther PM, Reedy J, Krebs-Smith SM. Development of the Healthy Eating Index-2005. J Am Diet Assoc 2008a;108(11):1896-901.

13 Andrade SC, Samantha C, Previdelli AN, Marchioni DML, Fisberg RM. Avaliação da confiabilidade e validade do Índice de Qualidade da Dieta Revisado. Rev Saude Publica 2013;41(1):5806-9. 
14 Guenther, PM, Reedy J, Krebs-Smith SM. Evaluation of the healthy eating index-2005. J Am Diet Assoc 2008b;108(11):1854-64.

15 Previdelli AN, Andrade SC, Pires MM, Ferreira SRG, Fisberg RM, Marchioni DC. Índice de Qualidade da Dieta Revisado para população brasileira. Rev Saúde Pública 2011;45(4):794-8.

16 Nascimento CM, Ribeiro AQ, Cotta RMM, Acurcio FA, Peixoto SV, Priore SE. Estado nutricional e fatores associados em idosos do Município de Viçosa, Minas Gerais, Brasil. Cad Saúde Pública 2011;27(12):2409-18.

17 Lwanga SK, Lemeshow S. Sample size determination in health studies. [Internet]. Geneva: WHO; 1991. [cited 2019 Jun 22] Available from: http://whqlibdoc.who.int/ publications/9241544058_(p1-p22).pdf

18 Fernandes DPS. Qualidade da dieta e fatores associados em idosos de Viçosa (MG): um estudo de base populacional. [dissertação]. Viçosa: Universidade Federal de Viçosa; 2016.

19 Pinheiro ABV, Lacerda EMA, Benzecry EH, Gomes MCS, Costa VM. Tabela para avaliação de consumo alimentar em medidas caseiras. 5a ed. São Paulo: Atheneu; 2005.

20 Araújo MOD, Guerra TMM. Alimentos "Per capita”. 3a ed. Natal: Editora da UFRN; 2007.

21 Previato HDRA, Barros FSS, Mello JBM, Silva FCS, Nimer M. Perfil clínico-nutricional e consumo alimentar de idosos do Programa Terceira Idade, Ouro Preto - MG. Demetra 2015;10(2):375-87.

22 Ferreira PM, Rosado GP. Perfil de usuários e percepção sobre a qualidade do atendimento nutricional em um programa de saúde para a terceira idade. RBGG 2012;15(2):243-54.

23 Ferreira MPN, Previdelli AN, Freitas TI, Marques KM, Goulart RMM, Aquino RC. Padrões dietéticos e fatores associados em idosos. RevBrasGeriatrGerontol. 2017;20(4):534-44.

24 Fernandes DPS, Duarte MSL, Pessoa MC. Healthy eating index: assessment of the diet quality of a brazilian elderly population. Nutr Metab Insights. 2018;1:1-7.
25 Nascimento FMP, Previdelli AN, Império FT, Maffei MK, Goulart RM, Monteiro ARC. Dietary patterns and associated factors among the elderly. Rev Bras GeriatrGerontol. 2017; 20(4):534-44.

26 Gomes AP, Soares ALG, Gonçalves H. Baixa qualidade da dieta de idosos: estudo de base populacional no sul do Brasil. Ciênc. Saúde Coletiva [Internet]. 2016 [cited 2019 Aug 23]; 21(11):3417-28. Available from: h t t p:// w w w. scielo.br/s c i e lo. php? script $=$ sci_arttext\&pid $=$ S 1413 $81232016001103417 \& \operatorname{lng}=$ en

27 Barbosa KGN. Condições de saúde bucal em idosos: uma revisão da realidade brasileira. OdontolClin. 2011;10(3):227- 31.

28 Ministério da Saúde - MS (BR). Saúde Bucal. Brasília: Ministério da Saúde; 2006.

29 Mourão LF, Xavier DAN, Neri AL, Luchesi KF. Estudo da associação entre doenças crônicas naturais do envelhecimento e alterações da deglutição referidas por idosos da comunidade. AudiolCommun Res. [Internet]. 2016 [citado 2019 Aug 23]; 21:1657. Disponível em: h t t p : / / w w w. s c i e 1 o.br/s c i e 1 o. php? script $=$ sci_arttext\&pid=S231764312016000100319\&lng=en

30 Wang AY, Sea MM, Ng K, Wang M, Chan IH, Lam CW, Sanderson JE, Woo J. Dietary fiber intake, myocardial injury, and major adverse cardiovascular events among end-stage kidney disease patients: a prospective cohort study. KidneyInt Rep. 2019 Mar 20;4(6):814-823. doi: 10.1016/j.ekir.2019.03.007.

31 Beckert F, Dallepiane LB, Kirchner RM, Silva FP, Merkoski TN, Silveira BC, Knob JI. Consumo de sal e hipertensão na população idosa: contribuição para o planejamento em saúde. REpS 2014;15(3):6-12.

32 Campos MTFS, Monteiro JBR, Ornelas APRC. Fatores que afetam o consumo alimentar e a nutrição do idoso. RevNut 2000;13(3):157-65.

33 Costa LML, Chagas DP, Marchi RJ, Neves HF, Balbinot HJ, Pereira PDM, Terezinha M. Healthy eating index in southern Brazilian older 
adults and its association with socioeconomic, behavioral and health characteristics. J Nutr Health Aging. 2012;16(1):3-7.

34 Malta MB, Papini SJ, Corrente JE. Avaliação da alimentação de idosos de município paulista Aplicação do Índice de Alimentação Saudável. CienSaudeColet. 2013;18:377-84.

35 Martins MPSC, Gomes ALM, Martins MCC, Mattos MA, Souza MDS Filho, Mello DB, et al. Consumo alimentar, pressão arterial e controle metabólico em idosos diabéticos hipertensos. RevBrasCard. 2010;23(3):162170.

36 Silva GM, Durante EB, Assumpção D, Barros MBA, Corona LP. Elevada prevalência de inadequação do consumo de fibras alimentares em idosos e fatores associados: um estudo de base populacional. RevBrasEpidemiol. 2019;22: E190044. doi: 10.1590/1980-549720190044.

37 Ministério da Saúde - MS (BR). Alimentação saudável para a pessoa idosa: um manual para profissionais de saúde. Brasília: Ministério da Saúde; 2009.

38 Richelsen B. Sugar-sweetened beverages and cardio-metabolic disease risks. CurrOpinClin NutrMetabCare. 2013;16:478-84.

39 Zanchim MC, Kirsten VR, Marchi ACB. Marcadores do consumo alimentar de pacientes diabéticos avaliados por meio de um aplicativo móvel. CienSaudeColet. 2018;23(12):41994208. [acessado em 2019 Jun 22]. Disponível em: http://dx.doi.org/10.1590/1413812320182312.01412017

40 Cesarino CB, Cipullo JP, Martin JFV, Ciorlia LA, Godoy MRP, Cordeiro JA, et al. Prevalência e fatores sócio demográficos em hipertensos de São José do Rio Preto - SP. Arq. Bras. Cardiol. 2008;91(1):31-5.

41 Sociedade Brasileira de Cardiologia. Diretrizes da Sociedade Brasileira de Cardiologia. [Internet]. Rio de Janeiro: Sociedade Brasileira de Cardiologia; 1943. [citado 2019 Ago 21]. Disponível em: http://publicacoes.cardiol. br/2014/img/pockets/POCKETBOOK_2017 interativa.pdf
42 Gadenz SD, Benvegnú LA. Hábitos alimentares na prevenção de doenças cardiovasculares e fatores associados em idosos hipertensos. CienSaudeColet. 2013;18(12):3523-34. 
